\title{
Invasive vegetation affects amphibian skin microbiota and body condition
}

Obed Hernández-Gómez ${ }^{\text {Corresp., }}{ }^{1}$, Allison Q Byrne ${ }^{1}$, Alex R Gunderson ${ }^{2}$, Thomas S Jenkinson ${ }^{1}$, Clay F Noss ${ }^{1}$, Andrew P Rothstein ${ }^{1}$, Molly C Womack ${ }^{1}$, Erica B Rosenblum ${ }^{1}$

1 Department of Environmental Science, Policy, and Management, University of California, Berkeley, Berkeley, United States

2 Department of Ecology and Evolutionary Biology, Tulane University, New Orleans, United States

Corresponding Author: Obed Hernández-Gómez

Email address: obedhg@berkeley.edu

Invasive plants are major drivers of habitat modification, and the scale of their impact is increasing globally as anthropogenic activities facilitate their spread. In California, an invasive plant genus of great concern is Eucalyptus. Eucalyptus leaves can alter soil chemistry and negatively affect underground macro- and microbial communities.

Amphibians serve as excellent models to evaluate the effect of Eucalyptus invasion on ground-dwelling species as they predate on soil arthropods and incorporate soil microbes into their microbiotas. The skin microbiota is particularly important to amphibian health, suggesting that invasive plant species could ultimately affect amphibian populations. To investigate the potential for invasive vegetation to induce changes in microbial communities, we sampled microbial communities in the soil and on the skin of local amphibians. Specifically, we compared Batrachoseps attenuatus skin microbiomes in both Eucalyptus globulus (Myrtaceae) and native Quercusagrifolia (Fagaceae) dominated forests in the San Francisco Bay Area. We determined whether changes in microbial diversity and composition in both soil and $B$. attenuatusskin were associated with dominant vegetation type. To evaluate animal health across vegetation types, we compared $B$. attenuatus body condition and the presence/absence of the amphibian skin pathogen Batrachochytrium dendrobatidis $(B d)$. We found that Eucalyptus invasion had no measurable effect on soil microbial community diversity and a relatively small effect (compared to the effect of site identity) on community structure in the microhabitats sampled.In contrast, our results show that $B$. attenuatus skin microbiota diversity was greater in Quercus dominated habitats. One amplicon sequence variant (ASV) identified in the family Chlamydiaceae was observed in higher relative abundance among salamanders sampled in Eucalyptus dominated habitats. We also observed that $B$. attenuatus body condition was higher in Quercus dominated habitats. Incidence of $B d$ across all individuals was very low (only one $B d$ positive individual). The effect on body condition demonstrates that although 
Eucalyptus may not always decrease amphibian abundance or diversity, it can potentially have cryptic negative effects. Our findings prompt further work to determine the mechanisms that lead to changes in the health and microbiome of native species postplant invasion. 
1 Invasive vegetation affects amphibian skin microbiota 2 and body condition

3

4

5

Obed Hernández-Gómez ${ }^{1}$, Allison Q. Byrne ${ }^{1}$, Alex R. Gunderson ${ }^{2}$, Thomas S. Jenkinson ${ }^{1}$, Clay F. Noss ${ }^{1}$, Andrew P. Rothstein ${ }^{1}$, Molly C. Womack ${ }^{1}$, Erica Bree Rosenblum ${ }^{1}$

${ }^{1}$ Department of Environmental Science, Policy, and Management, University of California, Berkeley, Berkeley, CA, United States

${ }^{2}$ Department of Ecology and Evolutionary Biology, Tulane University, New Orleans, LA, United States

\section{Corresponding Author:}

Obed Hernández-Gómez ${ }^{1}$

147 Hilgard Hall, Berkeley, CA, 94704, USA

Email address: obedhg@berkeley.edu

\section{Abstract}

Invasive plants are major drivers of habitat modification, and the scale of their impact is increasing globally as anthropogenic activities facilitate their spread. In California, an invasive plant genus of great concern is Eucalyptus. Eucalyptus leaves can alter soil chemistry and negatively affect underground macro- and microbial communities. Amphibians serve as excellent models to evaluate the effect of Eucalyptus invasion on ground-dwelling species as they predate on soil arthropods and incorporate soil microbes into their microbiotas. The skin microbiota is particularly important to amphibian health, suggesting that invasive plant species could ultimately affect amphibian populations. To investigate the potential for invasive vegetation to induce changes in microbial communities, we sampled microbial communities in the soil and on the skin of local amphibians. Specifically, we compared Batrachoseps attenuatus skin microbiomes in both Eucalyptus globulus (Myrtaceae) and native Quercus agrifolia (Fagaceae) dominated forests in the San Francisco Bay Area. We determined whether changes in microbial diversity and composition in both soil and $B$. attenuatus skin were associated with dominant vegetation type. To evaluate animal health across vegetation types, we compared $B$. attenuatus body condition and the presence/absence of the amphibian skin pathogen Batrachochytrium dendrobatidis $(B d)$. We found that Eucalyptus invasion had no measurable effect on soil microbial community diversity and a relatively small effect (compared to the effect of site identity) on community structure in the microhabitats sampled. In contrast, our results show that $B$. attenuatus skin microbiota diversity was greater in Quercus dominated habitats. One amplicon sequence variant (ASV) identified in the family Chlamydiaceae was observed in higher relative abundance among salamanders sampled in Eucalyptus dominated habitats. We also observed that $B$. attenuatus body condition was higher in Quercus dominated habitats. Incidence of $B d$ across all individuals was very low (only one $B d$ positive individual). The effect 
42 on body condition demonstrates that although Eucalyptus may not always decrease amphibian 43 abundance or diversity, it can potentially have cryptic negative effects. Our findings prompt 44 further work to determine the mechanisms that lead to changes in the health and microbiome of 45 native species post-plant invasion. 
46

47

48

49

50

51

52

53

54

55

56

57

58

59

60

61

62

63

64

65

66

67

68

69

70

\section{Introduction}

7

animal populations (Acevedo-Whitehouse \& Duffus 2009). Invasive plants are major drivers of

habitat modification, and the scale of their impacts is increasing globally (Pysek et al. 2012; van

Kleunen et al. 2015). In the United States, approximately 5,000 alien plants have been

introduced into natural ecosystems, causing significant ecological and environmental

degradation (Pimentel et al. 2005). Invasive plants are known to affect many patterns and

processes in native communities (e.g., changes in habitat structure, productivity, $\mathrm{pH}$,

transpiration, etc.), which in turn can have profound impacts on native species (Pysek et al.

2012; Vila et al. 2011). While the effect of invasive vegetation can vary based on the biology of

the plant and age of invasion (Hejda et al, 2009), changes in native community diversity and

composition have been documented in areas that have been dominated by invasive plants

(Batten et al, 2006; Tererai et al, 2013; Litt et al, 2014).

9 One way that invasive plants impact native animal populations is by altering their microbial commensals. Plant invasions could influence the microbial community structure of native fauna by changing microbial communities that hosts are exposed to, by altering host physiology, or both (Christian et al. 2015). Invasion may alter environmental microbial reservoirs

by shifting abiotic (e.g., temperature, moisture) and biotic (e.g., species diversity) conditions that affect the presence of certain microbial species (Batten et al. 2006; Coats \& Rumpho 2014).

However, the association between host microbiotas and the habitat microbial pool vary among studies. Among wild populations in relatively natural habitats, some studies have found that microbiomes vary significantly with habitat type (Bird et al. 2018; Bletz et al. 2017) whereas others have found that microbiomes are relatively conserved and coevolve with hosts (PradoIrwin et al. 2017). Thus, whether changes in the local microbial community structure also affect host microbial symbionts remains an open question. 
In California one of the invasive plants of greatest concern are the Eucalyptus sp. (Fork

72 et al. 2015; Wolf \& DiTomaso 2016). Eucalyptus were introduced into the state in the 1850's as

73 a timber species (Butterfield 1935), and multiple members of this genus are now abundant and

74 ecologically successful throughout the state (Ritter \& Yost 2009). Eucalyptus leaves can alter

75 soil nutrient availability (e.g., organic carbon, nitrogen, $\mathrm{O}_{2}$ ) resulting in changes in soil microbial

76 communities (Chen et al. 2013; Cortez et al. 2014). In addition, Eucalyptus leaf essential oils

77 have been observed to be toxic to soil fungi and negatively affect food palatability to soil

78 arthropods (Martins et al. 2013). Changes in toxicity and palatability can impact prey availability

79 for native fauna and subsequently may alter their microbiomes (Antwis et al. 2014). Resulting

80 changes in microbiomes may have important fitness consequences especially if microbial

81 species contribute to host physiological processes (Redford et al. 2012). Thus, Eucalyptus

82 invasions may alter the microbiome of native fauna by changing prey availability and/or shifting

83 the structure of microbial reservoirs.

Amphibians serve as excellent models to evaluate host-associated microbiome changes

in response to habitat changes as they predate on soil arthropods and incorporate soil microbes into their microbiotas (Loudon et al. 2014). The skin of amphibians is a vital organ used for respiration, osmoregulation, and immunity, but it is also sensitive to environmental changes, including temperature/moisture fluctuations, pollution, and infections (Brühl et al. 2011; Haslam et al. 2014). In addition, amphibian skin harbors diverse microbial communities that provide protection against lethal amphibian pathogens (Harris et al. 2009; Woodhams et al. 2014). Because the skin microbiota of amphibians recruits environmental microbes (Walke et al. 2014), environmental changes may result in consequential alterations to the amphibian skin community structure (Loudon et al. 2014; Muletz et al. 2012). Despite the importance of habitat quality in shaping amphibian skin microbiotas, only a handful of studies have evaluated the effect of environmental changes on these communities (Krynak et al, 2015; Costa et al, 2016; Krynak et al, 2016; Hughey et al, 2017), and, to our knowledge none have assessed the effect of invasive 
97 vegetation. The link between the skin microbiota and amphibian health suggests that

98 environmental changes like plant species invasions may negatively affect amphibian

99 populations.

100 To investigate potential changes induced by invasive vegetation on environmental and

101 host-associated microbial communities, we sampled microhabitat soil and Batrachoseps

102 attenuatus skin microbiomes in both Eucalyptus globulus (Myrtaceae) and native Quercus

103 agrifolia (Fagaceae) dominated forests in the San Francisco Bay Area. Specifically, we

104 determined whether changes in microbial composition, diversity, and stability in both soil and $B$.

105 attenuatus skin were associated with Eucalyptus or Quercus dominated habitat. To evaluate

106 animal health across Eucalyptus and Quercus dominated habitats, we also measured and

107 compared B. attenuatus body condition and the presence/absence of the amphibian chytrid

108 fungus Batrachochytrium dendrobatidis $(B d)$, which causes the lethal amphibian disease

109 chytridiomycosis. Our results illustrate a decline in the richness of skin associated microbiota

110 and a decrease in salamander body condition in Eucalyptus forest, illustrating that plant

111 invasions may have consequences for native species.

112 Materials \& Methods

113 Study System

114 The San Francisco Bay Area (Bay Area) provides an opportunity to test the effect of

115 Eucalyptus invasions on native fauna and their microbiotas. The Bay Area is home to numerous 116 seed-producing stands of E. globulus, E. pulchella, and E. viminalis among a mosaic of mixed

117 native evergreen forests and coastal scrublands. The discrete - yet interspersed - distribution

118 of invasive and native vegetation types in the Bay Area make it an ideal location to evaluate

119 vegetation effects on resident host-associated microbiotas while controlling for geography. In

120 addition, multiple ground dwelling amphibians are distributed throughout the Bay Area and may

121 be sensitive to effects of invasive Eucalyptus on the skin microbiome (Stebbins 2003). One

122 amphibian species that is present, abundant, and can be easily collected throughout invasive

Peer) reviewing PDF | (2019:10:42191:1:1:NEW 22 Dec 2019) 
123 Eucalyptus and native vegetation Bay Area habitats is Batrachoseps attenuatus. B. attenuatus

124 is an ideal focal species for testing the effects of invasive vegetation as it may be particularly

125 sensitive to local environmental changes (e.g., Eucalyptus invasion) because of their highly-

126 limited dispersal (<2 m lifetime movement; Maiorana 1978) and completely terrestrial life-cycle.

127 We sampled B. attenuatus between February 16 and March 1, 2018 within Quercus and

128 Eucalyptus dominant forest strands in Tilden Regional Park, Wildcat Canyon Regional Park, 129 and the University of California, Berkeley campus (Figure 1). To minimize non-vegetation

130 influences on the skin microbiota of $B$. attenuatus, we chose sites with similar elevation, slope,

131 and slope orientations previously described in Sax (2002, Table 1). We excluded one of the

132 native oak sites from Sax (2002; $\left.\mathrm{Native}_{3}\right)$ as it has been eroded by a nearby stream, and instead

133 chose a new site with similar characteristics.

\section{Field Methodology}

We handled all salamanders following a protocol approved by the University of

California, Berkeley Animal Care and Use Committee (protocol \# AUP-2015-01-7083-1). Access

137 to off-campus sampling sites was granted by East Bay Regional Parks under permit \# 965. We

collected salamanders by hand using gloves through log flip surveys within $\sim 100 \mathrm{~m}$ of the location coordinates. New gloves were donned between the handling of each salamander. To avoid resampling individuals and ensure that salamanders were later returned to their original capture site, we marked each capture log with the individual(s) identification number. We rinsed each salamander with $250 \mathrm{~mL}$ of sterile water and swabbed the dorsum with a sterile cotton swab 30 times. Following microbiota sampling, we measured each salamanders' total body

144 length and mass. All salamanders were returned to their location of capture immediately after 145 sampling. To characterize the microhabitat microbiota, we collected $\sim 20 \mathrm{mg}$ of soil or soil swabs

146 directly from under the logs where each salamander was captured after releasing each

147 individual. We collected 22 Batrachoseps attenuatus skin swabs from Quercus and 28 from 
148 Eucalyptus dominated habitats (Figure 1). All samples were stored on dry ice for up to 8 hours

149 and moved into a $-80 \mathrm{C}$ freezer upon return to the laboratory.

150 DNA Extraction, Amplification, and Sequencing

We isolated DNA from skin swab samples using the DNeasy PowerSoil DNA Isolation

152 Kit (Qiagen N.V., Hilden, Germany) following the modifications to the manufacturer's protocol

153 described in Hernández-Gómez et al. (2017b). Soil samples were processed similarly to swabs

154 for pre-cell lysis steps, but post-lysis steps were performed following the original manufacturer's

155 protocol to ensure proper removal of PCR inhibitors. To control for contamination, we included

156 unused swabs (i.e., negative extraction controls) and researcher glove swabs in our DNA

157 extractions. We amplified the bacterial 16S rRNA V4 region using primer pair F515/R806 with

158 the attachment of connector sequences that allows for the attachment of barcode/sequencing

159 adaptors (Hernandez-Gomez et al. 2017a). We ran each sample in triplicate, and each reaction

160 consisted of $5 \mu \mathrm{L}$ of template DNA, $7.5 \mu \mathrm{L}$ of $2 X$ MyTaq Master Mix (Bioline, Tauton, MA), 1.0

$161 \mu \mathrm{L}$ of $1 \mathrm{mM}$ forward and reverse primers, and $1.5 \mu \mathrm{L}$ of sterile water for a total of $15 \mu \mathrm{L}$ per

162 reaction. PCR conditions consisted of $94^{\circ} \mathrm{C}$ for 3 minutes, 30 cycles of $94^{\circ} \mathrm{C}$ for 45 seconds,

$16350^{\circ} \mathrm{C}$ for 60 seconds, and $72^{\circ} \mathrm{C}$ for 90 seconds, followed by $72^{\circ} \mathrm{C}$ for 10 minutes. We pooled

164 amplicon triplicates and cleaned the products using the UltraClean PCR Clean-up kit (Qiagen

165 N.V., Hilden, Germany).

166 We performed a second PCR on microbiota amplicons to ligate dual-index barcodes

167 paired with Illumina sequencing adaptors (Hernandez-Gomez et al. 2017a) to the ends of

168 amplicons. The PCR consisted of $5 \mu \mathrm{L}$ of clean amplicons, $7.5 \mu \mathrm{L} 2 \mathrm{X}$ MyTaq Master Mix, $1 \mu \mathrm{L}$

169 of $1 \mathrm{nM}$ forward and reverse barcode primers, and $1.5 \mu \mathrm{L}$ of water for a total of $15 \mu \mathrm{L}$ reactions.

170 PCR conditions consisted of $94^{\circ} \mathrm{C}$ for 3 minutes, 5 cycles of $94^{\circ} \mathrm{C}$ for 45 seconds, $65^{\circ} \mathrm{C}$ for 60

171 seconds, and $72^{\circ} \mathrm{C}$ for 90 seconds, followed by $72^{\circ} \mathrm{C}$ for 10 minutes. We quantified the PCR

172 products using a Qubit Fluorometer (Invitrogen Corp, Carlsbad, CA), pooled samples in

173 equimolar amounts, and cleaned the sample pool using the UltraClean PCR Clean-Up kit. The 
174 sample pool was submitted to the California Institute for Quantitative Biosciences Vincent J.

175 Coates Genomics Sequencing Laboratory to be sequenced on the MiSeq plaform (Illumina Inc.

176 San Diego, CA) using the Reagent Kit V3 to produce 300 bp paired end reads.

177 Bd detection and quantification

178 To detect and quantify $B d$ infection in our swabbed individuals, we performed real-time

179 quantitative PCR (qPCR) reactions in duplicate following Boyle et al. (2004) with slight

180 modifications. Each qPCR reaction consisted of $5 \mu \mathrm{L}$ of 1:10 diluted template DNA, $12.5 \mu \mathrm{L}$ of

181 2X TaqMan Fast Advanced Master Mix (Thermo Fisher, Waltham, MA), 900 nM forward/reverse

182 primers ITS1-3 Chytr and 5.8S Chytr (Boyle et al. 2004), 250 nM minor groove binder probe

183 Chytr MGB2 (Boyle et al. 2004), $400 \mathrm{ng} / \mu \mathrm{L}$ of BSA, and $2.75 \mu \mathrm{L}$ of molecular grade water for a

184 total reaction volume of $25 \mu \mathrm{L}$. For each 96-well qPCR reaction plate, we included three

185 replicates of a $B d$ zoospore standard dilution ranging from 100,000 to 0.1 genomic equivalents.

186 These zoospore standards were prepared using the Bd-GPL strain CJB7 - originally isolated

187 from Kings Canyon, California. At least three reactions per 96-well plate were designated as

188 negative controls, with each receiving $5 \mu \mathrm{L}$ of water in lieu of template DNA. To reduce the risk

189 of laboratory contamination we set up qPCR reactions in a laminar flow hood. We ran all qPCR

190 reactions on an Applied Biosystems StepOnePlus Real-Time PCR System (Thermo Fisher,

191 Waltham, MA), and used the manufacturer's software for standard curve analysis. We

192 considered an average qPCR quantification of less than one genomic equivalent per swab to be

193 negative for $B d$ infection.

194 Microbiota Sequence Analysis

195 We processed raw sequencing reads using Trimmomatic (Bolger et al. 2014) to remove

196 adapter sequences, bases below threshold quality of phred-20 from both ends of reads, and any

197 resulting reads under $30 \mathrm{bp}$. We paired reads that passed initial quality control using PANDAseq

198 (Masella et al. 2012). Only reads that paired successfully were employed in subsequent

199 analysis.

Peer) reviewing PDF | (2019:10:42191:1:1:NEW 22 Dec 2019) 
201 pipelines to filter erroneous reads, generate an amplicon sequence variant (ASV; error-

202 corrected unique DNA sequences) table, create a representative sequence phylogeny, and

203 assign taxonomy to ASVs. We chose to use ASVs rather than operational taxonomic units

204 (OTUs), because ASVs provide greater resolution in amplicon differentiation (Callahan et al.

205 2017). ASV variants can be denoted by single nucleotide differences based on sequencer error

206 correction methodologies, which surpass the accuracy obtained by OTU grouping which

207 implements an arbitrary sequence difference threshold to cluster amplicons. We processed the

208 resulting read file using the Quantitative Insights Into Microbial Ecology version 2.2018.4

209 (QIIME2) pipeline (Bolyen et al. 2018). We processed reads using the DADA2 plugin to quality

210 filter, dereplicate, remove chimeras and denoise reads using default settings (Callahan et al.

211 2016). We generated a phylogeny using MAFFT aligned representative ASV sequences using

212 default settings in FastTree2 to be used in alpha and beta analyses (Katoh \& Standley 2013;

213 Price et al. 2010). We applied a pre-trained Naïve Bayes classifier on the Greengenes 13_8

214 database to assign taxonomy to each ASV at the genus level (DeSantis et al. 2006). We ran the

215 ASV table through the package decontam in R to identify ASV's associated with glove

216 samples/negative extraction controls and removed identified contaminants from soil/swab ASV

217 table (Davis et al. 2017). In addition, we filtered out any ASV's whose taxonomy matched

218 chloroplast or mitochondria as these were not the target of our amplification protocol. To

219 standardize sequencing depth throughout all samples, we rarefied the filtered ASV table to

2205,115 sequences per sample. After filtering out 16S rRNA V4 amplicon sequence reads by base

221 pair quality and length, we processed 6,706,635 reads using QIIME2 to produce 16,176 ASV's

222 following contaminant and non-bacteria taxonomy removal. We deposited raw 16S rRNA V4

223 amplicon sequencing data into the NCBI Sequence Read Archive (project accession number

224 PRJNA574188). 

variables to evaluate normality prior to statistical model selection. We calculated three distinct alpha diversity metrics using the $\mathrm{R}$ packages vegan and picante: community richness (i.e., number of ASVs observed per sample), evenness (i.e., Shannon diversity indices), and phylogenetic diversity (i.e., Faith's phylogenetic diversity). We calculated these metrics in order to evaluate differences in the number of ASVs (community richness), distribution of ASV frequencies within samples (Shannon diversity index), and phylogenetic representation (Faith's phylogenetic diversity) across sample groups. To assess differences in community composition across samples, we applied the R packages GuniFrac and vegan to calculate three separate beta diversity metrics: pairwise unweighted/weighted UniFrac distances and Bray-Curtis dissimilarities. We chose to include these beta diversity metrics as they account for differences in presence/absence of phylogenetic lineages among samples (unweighted UniFrac), non-phylogenetic abundance-based differences among samples (Bray-Curtis). In addition, we computed the core microbiomes (i.e., ASVs shared among $70 \%$ of individuals) of Eucalyptus soil, Eucalyptus salamander skin, Quercus soil, and Quercus salamander skin samples.

Statistical Analysis evaluated differences in soil community diversity, composition, and community homogeneity between Quercus and Eucalyptus dominated habitats. To evaluate differences in alpha diversity between soil communities sampled in Quercus and Eucalyptus dominated habitats, we

247 implemented community richness, Shannon diversity indices, and phylogenetic diversity as 248 dependent variables, habitat type as a fixed variable, and site identity as a random variable in 249 linear mixed models ( $\mathrm{R}$ package Ime4; a negative binomial error distribution was used to 250 evaluate richness and Gaussian to evaluate Shannon diversity/phylogenetic diversity models). 
251 The significance of predictor variable was calculated with likelihood ratio tests (LRT). To

252 characterize the strength and significance of soil community compositional and structural

253 differentiation among habitat types, we implemented three PERMANOVA tests (R package

254 vegan) using weighted UniFrac, unweighted UniFrac, and Bray-Curtis dissimilarity matrices as

255 dependent variables and site identity as a random variable. We produced NMDS plots using the

256 three dissimilarity matrices to visualize clustering of samples by habitat and site identity using

257 the R package phyloseq. To assess whether habitat type influenced variation in soil community

258 structure, we performed three separate multivariate of homogeneity of group dispersions

259 analyses (R package vegan) using the beta diversity metrics. For each beta diversity metric, we

260 used a one-way ANOVA to test differences in point-to-centroid distances between soil samples

261 obtained from Eucalyptus and Quercus dominated habitats. We implemented an indicator

262 species analysis using the R package indicspecies to identify ASVs whose relative abundance

263 differs between soil samples collected in Eucalyptus and Quercus dominated habitats.

264

Effects of Eucalyptus invasion on B. attenuatus bacterial composition, diversity, and

265

stability: To assess whether salamander skin microbiota sampled in Quercus or Eucalyptus

266

dominated habitat differ in their overlap with microhabitat microbiota, we calculated average

267

unweighted UniFrac, weighted UniFrac, and Bray-Curtis dissimilarity values between every

268

salamander skin sample and all environmental samples in its corresponding site. We used

269

these dissimilarities as dependent variables in a generalized linear mixed model using a logit

270

distribution with habitat type as a fixed variable and site as a random variable. We also

271 evaluated whether skin microbial community diversity, composition, and heterogeneity differed

272 between B. attenuatus sampled in Quercus and Eucalyptus dominated habitats. We assessed

273 differences in community richness, Shannon diversity indices, and phylogenetic diversity

274 between salamander skin communities sampled in Quercus and Eucalyptus dominated habitats

275 using linear mixed models as described above. We ran PERMANOVA tests using weighted

276 UniFrac, unweighted UniFrac, and Bray-Curtis dissimilarities as dependent variables, habitat

Peer) reviewing PDF | (2019:10:42191:1:1:NEW 22 Dec 2019) 
277 type as a fixed variable, and site identity as a random variable to evaluate variation in 278 community composition and structure. We produced NMDS plots using the three dissimilarity 279 matrices to visualize clustering of skin samples by habitat and site identity. We also performed 280 three separate multivariate of homogeneity of group dispersions analyses to evaluate how 281 habitat type influenced variation in community structure among individuals in the same group. 282 For each beta diversity metric, we used a one-way ANOVA to test differences in point-to283 centroid distances between Eucalyptus and Quercus salamander skin microbial communities. 284 We implemented an indicator species analysis using the R package indicspecies to identify 285 ASVs whose relative abundance differs between salamander skin samples collected in 286 Eucalyptus and Quercus dominated habitats. Lastly, we assessed patterns of isolation by 287 distance (IBD) by comparing our three dissimilarity matrices of community composition to a 288 Euclidean geographic distance matrix between sampling locations. We tested for significance of 289 isolation by distance (IBD) through mantel tests using R package ade4 (Dray \& Siberchicot 290 2018).

Associations between Eucalyptus invasion and B. attenuatus body condition: We derived body condition indices for each salamander by obtaining the least squares regression residuals of mass and total body length. This methodology has been widely used to accurately evaluate estimates of body reserves in amphibians and other vertebrates (Ardia 2005; Schulte-Hostedde et al. 2005). We omitted two salamanders from the body condition analysis because they lost their tail immediately before or during sampling. We tested differences in body condition indices between individuals sampled in Eucalyptus and Quercus dominated habitats using a linear mixed model (R package Ime4) with site identity as a random factor. Lastly, we evaluated whether there were correlations between skin alpha diversity metrics (richness, Shannon

300 Diversity Index, and Faith's Phylogenetic Diversity) and body condition using Pearson 301 correlation tests. 


\section{Results}

303 Eucalyptus invasion has a small effect on microhabitat soil microbial community

304

305

306

307

308

309

310

311

312

313

314

315

316

317

318

319

320

321

322

323

324

325

326

327

\section{composition}

We found no differences in soil microbial alpha diversity (community richness: Likelihood Ratio Test, LRT = 0.086, $p=0.770 ;$ Shannon diversity index: $L R T=1 \times 10^{-4}, p=0.994$ phylogenetic diversity: LRT $=0.0071, p=0.933$; Figure $2 A-C)$. In contrast, habitat type explained a small proportion of the variation in community composition of soil samples (unweighted UniFrac: pseudo- $F_{1,30}=1.73, R^{2}=0.05, p=0.013$; weighted UniFrac: pseudo- $F_{1,30}$ $=2.38, R^{2}=0.07, p=0.021 ;$ Bray-Curtis: pseudo- $F_{1,30}=2.12, R^{2}=0.07, p=0.002 ;$ Figure $3 A-$ C). We did not observe significant differences in community heterogeneity among soil samples obtained from Eucalyptus and Quercus dominated habitats (unweighted UniFrac: pseudo- $\mathrm{F}_{1,30}=$ 3.21, $p=0.083$; weighted UniFrac: pseudo- $F_{1,30}=2.89, p=0.100 ;$ Bray-Curtis: pseudo- $F_{1,30}=$ $1.03, p=0.319)$. The core microbial communities of Eucalyptus dominated habitat soil samples (16 ASVs) were composed of ASV's characterized as Actinobacteria (two ASVs; average of $0.38 \%$ of reads across all samples) and Proteobacteria (14 ASVs; $5.91 \%$ ). In contrast, Quercus dominated habitat soil samples were composed of eight ASV's characterized as Actinobacteria (two ASVs; 0.37\%), Bacteroidetes (one ASVs; 0.19\%), Proteobacteria (four ASVs; $2.66 \%$ ), and Verrucomicrobia (one ASV; 0.93\%). The indicator species analysis identified 216 ASVs that differed significantly between habitat types with 122 associated with Eucalytpus soil samples and 94 associated with Quercus soil samples (Table S1). ASVs associated with either sample group were under a relative abundance of $1 \%$ indicating that only rare ASVs are influenced by cover type.

\section{B. attenuatus skin microbiota diversity is greater in native Quercus dominated habitats}

We did not observe differences in composition and structure between skin and corresponding soil samples among Quercus and Eucalyptus dominated habitats for unweighted UniFrac $(\mathrm{LTR}=0, \mathrm{p}=1.000$, mean $\pm \mathrm{SD}$ Quercus $=0.66 \pm 0.06$, mean \pm SD Eucalyptus $=0.73$ 
$328 \pm 0.08)$, weighted UniFrac (LRT $=0.673, p=0.412,0.32 \pm 0.11,0.39 \pm 0.11)$, or Bray-Curtis

329 dissimilarities $(\mathrm{LRT}=0, \mathrm{p}=1.000,0.86 \pm 0.09,0.94 \pm 0.05)$. Microbial community richness and

330 phylogenetic diversity were significantly lower for salamanders in Eucalyptus dominated habitat

331 compared to salamanders sampled in Quercus habitats (community richness: LRT $=4.07, p=$

332 0.044; Shannon diversity index: $L R T=1.75, p=0.185 ;$ phylogenetic diversity: $L R T=4.43, p=$

3330.035 ; Figure 2D-F). Habitat type significantly explained a small portion of the differentiation in

334 the skin composition and structure of $B$. attenuatus (unweighted UniFrac: pseudo- $F_{1,48}=2.48$,

$335 R^{2}=0.05, p=0.001$; weighted UniFrac: pseudo- $F_{1,48}=2.64, R^{2}=0.05, p=0.031$; Bray-Curtis:

336 pseudo- $F_{1,48}=2.61, R^{2}=0.05, p=0.004$; Figure 3D-F). We noted significantly higher

337 heterogeneity among $B$. attenuatus skin microbiotas from Eucalyptus dominated habitat than

338 Quercus dominated habitat for UniFrac metrics (unweighted UniFrac: pseudo- $F_{1,48}=4.13, p=$

339 0.048; weighted UniFrac: pseudo- $\left.F_{1,48}=12.75, p<0.001\right)$ but not for Bray-Curtis dissimilarities

340 (pseudo- $F_{1,48}=0.295, p=0.590 ;$ Figure 4). We found a significant association with geographic

341 distance and our Bray-Curtis dissimilarity (Mantel Bray-Curtis: $r=0.17, p=0.017$ ) but no

342 patterns of isolation by distance with both our weighted and unweighted UniFrac matrices

343 (Mantel unweighted UniFrac: $r=-0.04, p=0.581$; weighted UniFrac: $r=0.08, p=0.152$ ).

344 The core microbiota of salamander skin samples collected in Eucalyptus dominated

345 habitat was comprised entirely of ASVs assigned to the phylum Proteobacteria (eight ASVs;

$34632.27 \%$ of reads per sample). The core microbiota of salamander skin samples collected in

347 Quercus dominated habitat was richer in that it possessed 32 ASVs assigned to the phyla

348 Actinobacteria (12 ASVs; 2.29\%), Chloroflexi (1 ASV; 0.19\%), Proteobacteria (17 ASVs;

$34925.29 \%$ ), and Verrucomicrobia (2 ASVs; $0.50 \%$ ). As observed in the soil core microbial

350 communities, salamander skin core ASVs consisted mostly of rare taxa (i.e., less than 1\%).

351 However, we found one skin ASV identified as Bordetella petrii to dominate skin communities in

352 Eucalyptus (29.81\%) and Quercus (20.45\%) dominated habitats. The indicator species analysis 
353 identified 294 ASVs that differed significantly between habitat types with 36 associated with

354 Eucalyptus salamander skin samples and 258 associated with Quercus salamander skin

355 samples (Table S2). As observed in the soil sample indicator analysis, a majority of associated

356 ASVs were rare (relative abundance $<1 \%$ ). However, one ASV identified to the family

357 Chlamydiaceae was significantly associated with and abundant in salamanders sampled from

358 Eucalyptus dominated habitats (relative abundance mean \pm SD: $4.32 \% \pm 9.95 \%$, range: 0 -

$35944.61 \%)$ compared to individuals collected in Quercus dominated habitats $\left(8.89 \times 10^{-5} \% \pm 2.94\right.$

$\left.360 \times 10^{-4 \%}, 0-1.17 \times 10^{-3} \%\right)$.

361 B. attenuatus body condition is higher in native Quercus dominated habitat

$362 \quad$ Body condition indices differed significantly between salamanders sampled in

363 Eucalyptus and Quercus dominated habitats $(\mathrm{LTR}=5.38, \mathrm{p}=0.020)$. Salamanders sampled in

364 Quercus dominated habitat possessed higher body condition indices than those sampled from

365 the Eucalyptus dominated habitat (Figure 5). We did not find significant correlations between

366 body condition indices and skin microbial community richness $(R=0.13, p=0.385)$, Shannon

367 Diversity Index $(R=0.13, p=0.359)$, or Faith's phylogenetic diversity $(R=0.15, p=0.297)$. One

368 salamander (OHG-47_S202) tested positive for $B d$ with a low average infection load of 41.59

369 zoospore equivalents (Supplementary Table S1). The prevalence of infection in our study sites

370 overall was $2.0 \%$ (1/50), and prevalence did not vary significantly between our Quercus and

371 Eucalyptus dominated sites $(\mathrm{t}=-1, \mathrm{df}=20, \mathrm{p}=0.329)$.

\section{Discussion}

We observed no differences in richness, little variation in community composition and

374 structure, and similar differentiation between soil and skin samples across Eucalyptus and

375 Quercus dominated sites. Thus, the filter acting on microbial richness is likely operating at the

376 host level, rather than the passive uptake of different soil microbial communities. At the

377 individual level, we found differences in native salamander skin microbial diversity and body 
378

379

380

381

382

383

384

condition associated with Eucalyptus invasion. We also observed higher salamander skin microbial composition heterogeneity and relative abundances of an ASV identified to the family Chlamydiaceae in salamanders inhabiting Eucalyptus dominated habitats.

\section{Effects of invasion on the local environment's microbial community}

We found no differences in soil microbiome diversity between native and Eucalyptus sites. In addition, a small fraction of the variation in community composition among soil samples was explained by vegetation type. This finding is surprising given other studies documenting shifts in soil microbiomes following plant invasions (Zhang et al. 2018) and the unique chemical properties of Eucalyptus leaf litter (Martins et al. 2013). The small differences observed could be driven by an inability to capture the entire microbial community with $16 \mathrm{~S}$ sequencing. For example, we did not measure fungal diversity, a portion of the soil microbiome that has been shown to vary by space and dominant vegetation (Sterkenburg et al. 2015). In addition, lack of variation in soil microbiota among habitats could be driven by conservation of microbial communities in microhabitats. For example, our soil samples were all collected under logs (where the salamanders were found), which could buffer the soil from an accumulation of Eucalyptus leaf litter, desiccation, and bacteria dispersal from forest floor microbiota (Mäkipää et al. 2017).

Differences in skin microbial alpha diversity of B. attenuatus associated with Eucalyptus invasion

We found no difference between the two habitats in the overlap between salamander skin and the local soil. In both habitats, the dissimilarities between the skin microbial communities and those in the environment reservoirs are similar. These observations suggest that the filter acting on microbial richness is most likely operating at the host level rather than at the host-environment interface.

Microbial richness was higher in skin swab samples collected from salamanders in native Quercus habitat than in those collected in Eucalyptus habitat. This difference might be 
404 driven by the dropout of rare taxa - or the proliferation of already abundant members of the 405 microbiota (e.g., Chlamydiaceae) - in the Eucalyptus samples. While we cannot differentiate 406 among these two possibilities using $16 \mathrm{~S}$ rRNA amplicon sequencing alone, this pattern could 407 reflect microbial community changes due to environmental filters present in the Eucalyptus 408 dominated habitat. Changes in the relative abundance of core bacteria and alpha diversity have 409 been documented in previous studies assessing the effects of environmental changes (e.g. 410 captivity and changes in environmental reservoirs) on the skin microbiota of amphibians (Becker 411 et al. 2014; Wuerthner et al., in press). Soils in Eucalyptus dominated plots can be a harsh 412 environment for certain plants and microbes due to allelopathic chemicals leeched via leaves 413 and roots, increase of soil water repellency, and changes to soil chemistry (Behera \& Sahani 414 2003; Dellacassa et al. 1989; Ruwanza et al. 2015). This harsh soil environment could exclude 415 some taxa that are rare in the soil, but abundant on the skin of amphibians (Walke et al. 2014). 416 Although we did not detect differences in soil microbial alpha diversity between Quercus and 417 Eucalyptus soils, a negative effect of Eucalyptus on salamander-associated bacteria is still 418 possible. While a direct link between amphibian skin microbial community diversity and skin 419 health (e.g., resistance to the pathogen $B d$ ) has not been established (Jimenez and Sommer 420 2016), richer communities can possess greater functional diversity that may enhance the 421 protective function of the skin microbiome (Hernández-Gómez et al, 2019). As such, we 422 recommend that future studies characterize the functionality of amphibian skin communities in 423 the context of plant invasions to determine whether relevant microbial functions are affected as 424 well.

425 Differences in community heterogeneity and relative abundance of Chlamydiaceae in $B$. 426 attenuatus skin microbiota associated with Eucalyptus invasion

427 We did not observe clear differences in microbial community composition on the skin of 428 salamanders found in native oak compared to Eucalyptus habitat. Similar microbial composition 429 across habitats could be driven by a number of factors related to the assembly of these 
430 communities such as: geographic distance among sites, microhabitat environment

431 characteristics, and strong associations between $B$. attenuatus and members of the core

432 microbiota. For geographic distance, we found that Bray-Curtis dissimilarity matrices were

433 significantly correlated with distance between sites, indicating that patterns of isolation by

434 distance may be an important driver of observed variation and may supersede large-scale

435 environmental filters on abundant bacteria (e.g. vegetation type, elevation, slope). Additionally,

436 Batrachoseps attenuatus' high site fidelity, relatively short dispersal distances, and association

437 with fallen logs may decrease differences compared to surrounding leaf litter, which may show

438 greater microbial composition differences between sites (Welsh Jr \& Droege 2001). This result

439 is in contrast to similar studies of Salamandra salamandra, where individuals residing in

440 different habitats (ponds versus streams) possess distinct skin microbial communities (Sanchez

441 et al. 2017). Finally, given the potential of microbiomes to influence individual fitness,

442 salamanders' microbes could be under selection for retaining certain microbial profiles

443 regardless of environment. For example, Kueneman et al., (2014) identified species specific

444 skin microbiotas among co-habiting amphibians across distinct habitats, suggesting a strong

445 association between amphibian species and certain microbial skin symbionts. Therefore, there

446 may be a skin microbiome profile for Batrachoseps sp. that is consistent across potential

447 habitats given their broad geographic distribution.

448 Despite overlap in community composition among B. attenuatus in Eucalyptus and

449 Quercus dominated habitats, we noted greater phylogenetic heterogeneity in the skin microbiota

450 of Eucalyptus inhabiting salamanders than in that of native Quercus residents. While we

451 controlled for the effect of geography, altitude, and slope among our sites, it is possible that

452 differences in microhabitat abiotic (e.g., soil chemistry) and biotic factors (e.g., understory

453 vegetation, forest stand age) may have disproportionate effects on the persistence of distinct

454 rare bacteria in these salamanders. The loss of bacterial species in salamanders inhabiting

455 Eucalyptus habitat may vary by individual- and site-specific factors that were not measured in

Peer) reviewing PDF | (2019:10:42191:1:1:NEW 22 Dec 2019) 
456 the current study. Heterogeneous responses of microbial communities have been demonstrated

457 for soil microbes in response to silviculture and agricultural practices (Degrune et al. 2017).

458 Similarly for host-associated microbiotas, variability in beta diversity has been observed for

459 microbial symbiont communities in response to environmental stressors (e.g., increased

460 temperature, acidification, pollution; Zaneveld et al. 2017).

461 Despite an absence of differentiation in skin microbial community composition and

462 structure between salamanders sampled in Eucalyptus and Quercus dominant habitats, we did

463 observe a high relative abundance and almost exclusive presence of an ASV identified to the

464 family Chlamydiaceae in Eucalyptus salamander skin. This single ASV difference is significant,

465 as members of the family Chlamydiaceae have been identified as potential salamander

466 pathogens (Martel et al. 2012). Systematic infection with Chlamydiaceae pathogens in

467 salamanders has been documented to result in anorexia, lethargy, edema, abnormal gate, and

468 death (Martel et al. 2012). Although we did not observe any signs of disease in the $B$.

469 attenuatus sampled in Eucalyptus dominated habitats (other than lower body condition indices),

470 it is possible that an increase in Chlamydiaceae in the skin of salamanders results from

471 microbial dysbiosis (Prado-Irwin et al, 2017). Other skin microbiota studies on terrestrial

472 salamanders in the San Francisco Bay Area have identified Chlamydiaceae taxa on the skin

473 microbial communities; however, the relative abundances of this taxa are usually rare as we

474 observed in salamanders inhabiting Quercus habitat (Bird et al, 2018). While we cannot

475 effectively link the increase in Chlamydiaceae to infectious disease in our salamanders, it is

476 important that future studies on terrestrial salamanders evaluate whether this bacterial group

477 poses a threat to the health of amphibians in disturbed habitats.

\section{Potential effects of Eucalyptus invasion on $B$. attenuatus body condition}

479 We found that the body condition index of salamanders in Eucalyptus forest was

480 significantly lower than those found in Quercus woodlands. The effect on body condition is

481 consistent with prior work demonstrating a negative effect of introduced Eucalyptus on 
482 amphibian diversity (Fork et al. 2015; Russell \& Downs 2012). Importantly, our results also 483 suggest that although Eucalyptus may not always decrease amphibian abundance or diversity 484 (e.g. Keane \& Morrison 1990; Sax 2002), it can have more cryptic negative effects. Lower body 485 condition means that $B$. attenuatus in Eucalyptus forest may have less energy reserves (and 486 potentially lower fitness) than those in native Quercus woodland (Schulte-Hostedde et al. 2005). 487 In our study, body condition was not correlated with specific skin microbiome characteristics. 488 Although we cannot determine what proximate factor is driving body condition decline in 489 Eucalyptus habitat, there are multiple possible explanations including decreased prey 490 availability. For example, Fork et al. (2015) found lower arthropod richness and lower 491 abundance of some arthropod classes in Eucalyptus relative to oak woodland habitat (although 492 an earlier study did not find differences in leaf litter arthropod richness in Eucalyptus versus 493 Quercus woodlands Sax (2002). In addition, Eucalyptus leaf extract has been found to compromise chemical communication in salamanders, and this could contribute to stress in Batrachoseps residing in invasive vegetation dominated habitat (Iglesias-Carrasco et al. 2017). Ultimately, a more comprehensive comparison of abiotic and biotic characteristics in relevant microhabitats is needed to fully understand the effects of invasive vegetation on terrestrial salamanders.

\section{Conclusions}

500 To our knowledge, our study is the first to demonstrate changes in the microbial communities of native hosts associated with plant invasion (Eucalyptus). Interestingly, we found differences in the skin microbial community of the native salamander $B$. attenuatus, but no differences in soil microbial communities between Eucalyptus and native oak habitats. We also

504 found decreased body condition of this native salamander in Eucalyptus dominated habitats.

505 Our findings prompt further experimental work to determine the mechanisms causing these 506 microbial changes and their potential effect on the fitness of native fauna following invasion. 


\section{Acknowledgements}

508 We thank Shannon Buttimer and Natasha Septanova for their help in field collections.

509 We also thank Albert Tang, Carissa Tinoco, and Shannon Buttimer for their help in the

510 laboratory. We are grateful to Andrea Jani and one anonymous reviewer for their comments and

511 suggestions to the text.

512 


\section{References}

514 Acevedo-Whitehouse K, and Duffus ALJ. 2009. Effects of environmental change on wildlife

515

516

517

518

519

520

521

522

523

524

525

526

527

528

529

530

531

532

533

534

535

536

537

538

539

540

541

542

543

544

545

546

547

548

549

550

551

552

553

554

555

556

557

558

559

560

561 health. Philosophical Transactions of the Royal Society B-Biological Sciences 364:34293438 DOI: $10.1098 /$ rstb.2009.0128.

Antwis RE, Haworth RL, Engelmoer DJP, Ogilvy V, Fidgett AL, and Preziosi RF. 2014. Ex-situ Diet Influences the Bacterial Community Associated with the Skin of Red-Eyed Tree Frogs (Agalychnis callidryas). Plos One 9 DOI: 10.1371/journal.pone.0085563.

Ardia D. 2005. Super size me: an experimental test of the factors affecting lipid content and the ability of residual body mass to predict lipid stores in nestling European Starlings. Functional Ecology 19:414-420.

Batten KM, Scow KM, Davies KF, and Harrison SP. 2006. Two invasive plants alter soil microbial community composition in serpentine grasslands. Biological Invasions 8:217230 DOI: 10.1007/s10530-004-3856-8.

Becker MH, Richards-Zawacki CL, Gratwicke B, and Belden LK. 2014. The effect of captivity on the cutaneous bacterial community of the critically endangered Panamanian golden frog (Atelopus zeteki). Biological Conservation 176:199-206 DOI: 10.1016/j.biocon.2014.05.029.

Behera N, and Sahani U. 2003. Soil microbial biomass and activity in response to Eucalyptus plantation and natural regeneration on tropical soil. Forest Ecology and Management 174:1-11.

Bird AK, Prado-Irwin SR, Vredenburg VT, and Zink AG. 2018. Skin microbiomes of California terrestrial salamanders are influenced by habitat more than host phylogeny. Frontiers in Microbiology 9 DOI: 10.3389/fmicb.2018.00442.

Bletz MC, Archer H, Harris RN, McKenzie VJ, Rabemananjara FCE, Rakotoarison A, and Vences M. 2017. Host ecology rather than host phylogeny drives amphibian skin microbial community structure in the biodiversity hotspot of Madagascar. Frontiers in Microbiology 8:1-14 DOI: 10.3389/fmicb.2017.01530.

Bolger AM, Lohse M, and Usadel B. 2014. Trimmomatic: a flexible trimmer for Illumina sequence data. Bioinformatics 30:2114-2120 DOI: 10.1093/bioinformatics/btu170.

Bolyen E, Rideout JR, Dillon MR, Bokulich NA, Abnet C, Al-Ghalith GA, Alexander H, Alm EJ, Arumugam M, and Asnicar F. 2018. QIIME 2: Reproducible, interactive, scalable, and extensible microbiome data science. PeerJ Preprints 6:e27295v2.

Boyle D, Boyle D, Olsen V, Morgan J, and Hyatt A. 2004. Rapid quantitative detection of chytridiomycosis (Batrachochytrium dendrobatidis) in amphibian samples using real-time Taqman PCR assay. Diseases of Aquatic Organisms 60:141-148.

Brühl CA, Pieper S, and Weber B. 2011. Amphibians at risk? Susceptibility of terrestrial amphibian life stages to pesticides. Environmental Toxicology and Chemistry 30:24652472.

Butterfield H. 1935. The introduction of Eucalyptus into California. Madrono 3:149-154.

Callahan BJ, McMurdie PJ, and Holmes SP. 2017. Exact sequence variants should replace operational taxonomic units in marker-gene data analysis. ISME Journal 11:2639-2643 DOI: 10.1038/ismej.2017.119.

Callahan BJ, McMurdie PJ, Rosen MJ, Han AW, Johnson AJA, and Holmes SP. 2016. DADA2: High-resolution sample inference from Illumina amplicon data. Nature Methods 13:581 DOI: $10.1038 / n m e t h .3869$.

Chen FL, Zheng H, Zhang K, Ouyang ZY, Lan J, Li HL, and Shi Q. 2013. Changes in soil microbial community structure and metabolic activity following conversion from native Pinus massoniana plantations to exotic Eucalyptus plantations. Forest Ecology and Management 291:65-72 DOI: 10.1016/j.foreco.2012.11.016.

Peer] reviewing PDF | (2019:10:42191:1:1:NEW 22 Dec 2019) 
562

563

564

565

566

567

568

569

570

571

572

573

574

575

576

577

578

579

580

581

582

583

584

585

586

587

588

589

590

591

592

593

594

595

596

597

598

599

600

601

602

603

604

605

606

607

608

609

610

Christian N, Whitaker BK, and Clay K. 2015. Microbiomes: unifying animal and plant systems through the lens of community ecology theory. Frontiers in Microbiology 6 DOI: 10.3389/fmicb.2015.00.869.

Coats VC, and Rumpho ME. 2014. The rhizosphere microbiota of plant invaders: an overview of recent advances in the $\mathrm{m}$ crobiomics of invasive plants. Frontiers in Microbiology 5 DOI: 10.3389/fmicb.2014.00368.

Cortez CT, Nunes L, Rodrigues LB, Eisenhauer N, and Araujo ASF. 2014. Soil microbial properties in Eucalyptus grandis plantations of different ages. Journal of Soil Science and Plant Nutrition 14:734-742.

Costa S, Lopes I, Proença DN, Ribeiro R, and Morais PV. 2016. Diversity of cutaneous microbiome of Pelophylax perezi populations inhabiting different environments. Science of the Total Environment 572:995-1004.

Davis NM, Proctor D, Holmes SP, Relman DA, and Callahan BJ. 2017. Simple statistical identification and removal of contaminant sequences in marker-gene and metagenomics data. bioRxiv DOI: 10.1101/221499.

Degrune F, Theodorakopoulos N, Colinet G, Hiel M-P, Bodson B, Taminiau B, Daube G, Vandenbol M, and Hartmann M. 2017. Temporal dynamics of soil microbial communities below the seedbed under two contrasting tillage regimes. Frontiers in Microbiology $8: 1127$.

Dellacassa E, Menendez P, Moyna P, and Cerdeiras P. 1989. Antimicrobial activity of Eucalyptus essential oils. Fitoterapia 60:544-546.

DeSantis TZ, Hugenholtz P, Larsen N, Rojas M, Brodie EL, Keller K, Huber T, Dalevi D, Hu P, and Andersen GL. 2006. Greengenes, a chimera-checked 16S rRNA gene database and workbench compatible with ARB. Applied and Environmental Microbiology 72:50695072 DOI: 10.1128/aem.03006-05.

Dray S, and Siberchicot MA. 2018. Package 'ade4'.

Fork S, Woolfolk A, Akhavan A, Van Dyke E, Murphy S, Candilor B, Newberry T, Schreibman S, Salisbury J, and Wasson K. 2015. Biodiversity effects and rates of spread of nonnative eucalypt woodlands in central California. Ecological Applications 25:2306-2319 DOI: 10.1890/14-1943.1.sm.

Harris RN, Brucker RM, Walke JB, Becker MH, Schwantes CR, Flaherty DC, Lam BA, Woodhams DC, Briggs CJ, Vredenburg VT, and Minbiole KPC. 2009. Skin microbes on frogs prevent morbidity and mortality caused by a lethal skin fungus. ISME Journal 3:818-824 DOI: 10.1038/ismej.2009.27.

Haslam IS, Roubos EW, Mangoni ML, Yoshizato K, Vaudry H, Kloepper JE, Pattwell DM, Maderson PFA, and Paus R. 2014. From frog integument to human skin: dermatological perspectives from frog skin biology. Biological Reviews 89:618-655 DOI: 10.1111/brv.12072.

Hejda M, Pyšek P, and Jarošík V. 2009. Impact of invasive plants on the species richness, diversity and composition of invaded communities. Journal of Ecology 97:393-403.

Hernández-Gómez O, Hoverman JT, and Williams RN. 2017a. Cutaneous microbial community variation across populations of eastern hellbenders (Cryptobranchus alleganiensis alleganiensis). Frontiers in Microbiology 8 DOI: 10.3389/fmicb.2017.01379.

Hernández-Gómez O, Kimble SJA, Briggler JT, and Williams RN. 2017b. Characterization of the cutaneous bacterial communities of two giant salamander subspecies. Microbial Ecology 73:445-454 DOI: 10.1007/s00248-016-0859-9.

Hernández-Gómez O, Briggler JT, and Williams RN. 2019. Captivity-induced changes in the skin microbial communities of hellbenders (Cryptobranchus alleganiensis). Microbial Ecology 77:782-793.

Peer] reviewing PDF | (2019:10:42191:1:1:NEW 22 Dec 2019) 
611 Hughey MC, Pena JA, Reyes R, Medina D, Belden LK, and Burrowes PA. 2017. Skin bacterial

612

613

614

615

616

617

618

619

620

621

622

623

624

625

626

627

628

629

630

631

632

633

634

635

636

637

638

639

640

641

642

643

644

645

646

647

648

649

650

651

652

653

654

655

656

657

658

659

660

661 microbiome of a generalist Puerto Rican frog varies along elevation and land use gradients. PeerJ DOI:107717/peerj.3688

Iglesias-Carrasco M, Head ML, Jennions MD, Martín J, and Cabido C. 2017. Leaf extracts from an exotic tree affect responses to chemical cues in the palmate newt, Lissotriton helveticus. Animal Behaviour 127:243-251.

Jimenez RR, and Sommer S. 2016. The amphibian microbiome: natural range of variation, pathogenic dysbiosis, and role in conservation. Biodiversity and Conservation 26:763786.

Katoh K, and Standley DM. 2013. MAFFT Multiple Sequence Alignment Software Version 7: improvements in performance and usability. Molecular Biology and Evolution 30:772-780 DOI: $10.1093 / \mathrm{molbev} / \mathrm{mst0} 10$.

Keane JJ, and Morrison ML. 1990. Comparative use of eucalyptus versus native vegetation by wildlife on Angel Island State Park, California. Transactions of the Western Section of the Wildlife Society (USA).

Kueneman JG, Parfrey LW, Woodhams DC, Archer HM, Knight R, and McKenzie VJ. 2014. The amphibian skin-associated microbiome across species, space and life history stages. Molecular Ecology 23:1238-1250 DOI: 10.1111/mec.12510.

Krynak KL, Burke DJ, and Benard MF. 2015. Larval environment alters amphibian immune defenses differentially across life stages and populations. PlosOne DOI: 10.1371/journal.pone.0130383

Krynak KL, Burke DJ, and Benard MF. 2016. Landscape and water characteristics correlate with immune defense traits across Blanchard's cricket frog (Acris blanchardi) populations. Biological Conservation 193:153-167.

Litt AR, Cord EE, Fulbright TE, and Shuster GL. 2014. Effects of invasive plants on arthropods. Conservation Biology 28:1532-1549.

Loudon AH, Woodhams DC, Parfrey LW, Archer H, Knight R, McKenzie V, and Harris RN. 2014. Microbial community dynamics and effect of environmental microbial reservoirs on red-backed salamanders (Plethodon cinereus). ISME Journal 8:830-840 DOI: 10.1038/ismej.2013.200.

Maiorana VC. 1978. Behavior of an unobservable species: diet selection by a salamander. Copeia:664-672.

Martel A, Adriaensen C, Bogaerts S, Ducatelle R, Favoreel H, Crameri S, Hyatt AD, Haesebrouck F, and Pasmans F. 2012. Novel Chlamydiaceae disease in captive salamanders. Emerging Infectious Diseases 18:1020-1022.

Martins C, Natal-da-Luz T, Sousa JP, Goncalves MJ, Salgueiro L, and Canhoto C. 2013. Effects of essential oils from Eucalyptus globulus leaves on soil organisms involved in leaf degradation. Plos One 8 DOI: 10.1371/journal.pone.0061233.

Masella AP, Bartram AK, Truszkowski JM, Brown DG, and Neufeld JD. 2012. PANDAseq: PAired-eND Assembler for Illumina sequences. BMC Bioinformatics 13 DOI: 10.1186/1471-2105-13-31.

Muletz CR, Myers JM, Domangue RJ, Herrick JB, and Harris RN. 2012. Soil bioaugmentation with amphibian cutaneous bacteria protects amphibian hosts from infection by Batrachochytrium dendrobatidis. Biological Conservation 152:119-126 DOI: 10.1016/j.biocon.2012.03.022.

Mäkipää R, Rajala T, Schigel D, Rinne KT, Pennanen T, Abrego N, and Ovaskainen O. 2017. Interactions between soil-and dead wood-inhabiting fungal communities during the decay of Norway spruce logs. ISME Journal 11:1964.

Pimentel D, Zuniga R, and Morrison D. 2005. Update on the environmental and economic costs associated with alien-invasive species in the United States. Ecological Economics 52:273-288 DOI: 10.1016/j.ecolecon.2004.10.002.

Peer) reviewing PDF | (2019:10:42191:1:1:NEW 22 Dec 2019) 
662

663

664

665

666

667

668

669

670

671

672

673

674

675

676

677

678

679

680

681

682

683

684

685

686

687

688

689

690

691

692

693

694

695

696

697

698

699

700

701

702

703

704

705

706

707

708

709

710

Prado-Irwin SR, Bird AK, Zink AG, and Vredenburg VT. 2017. Intraspecific variation in the skinassociated microbiome of a terrestrial salamander. Microbial Ecology 74:745-756 DOI: 10.1007/s00248-017-0986-y.

Price MN, Dehal PS, and Arkin AP. 2010. FastTree 2-Approximately Maximum-Likelihood Trees for Large Alignments. Plos One 5 DOI: 10.1371/journal.pone.0009490.

Pysek P, Jarosik V, Hulme PE, Pergl J, Hejda M, Schaffner U, and Vila M. 2012. A global assessment of invasive plant impacts on resident species, communities and ecosystems: the interaction of impact measures, invading species' traits and environment. Global Change Biology 18:1725-1737 DOI: 10.1111/j.13652486.2011.02636.x.

Redford KH, Segre JA, Salafsky N, del Rio CM, and McAloose D. 2012. Conservation and the Microbiome. Conservation Biology 26:195-197 DOI: 10.1111/j.1523-1739.2012.01829.x.

Ritter M, and Yost J. 2009. Diversity, reproduction, and potential for invasiveness of Eucalyptus in California. Madroño 56:155-168.

Russell C, and Downs CT. 2012. Effect of land use on anuran species composition in northeastern KwaZulu-Natal, South Africa. Applied Geography 35:247-256.

Ruwanza S, Gaertner M, Esler KJ, and Richardson DM. 2015. Allelopathic effects of invasive Eucalyptus camaldulensis on germination and early growth of four native species in the Western Cape, South Africa. Southern Forests: a Journal of Forest Science 77:91-105.

Sanchez E, Bletz MC, Duntsch L, Bhuju S, Geffers R, Jarek M, Dohrmann AB, Tebbe CC, Steinfartz S, and Vences M. 2017. Cutaneous bacterial communities of a poisonous salamander: a perspective from life stages, body parts and environmental conditions. Microbial Ecology 73:455-465.

Sax DF. 2002. Equal diversity in disparate species assemblages: a comparison of native and exotic woodlands in California. Global Ecology and Biogeography 11:49-57 DOI: 10.1046/j.1466-822X.2001.00262.x.

Schulte-Hostedde AI, Zinner B, Millar JS, and Hickling GJ. 2005. Restitution of mass-size residuals: validating body condition indices. Ecology 86:155-163.

Stebbins RC. 2003. A field guide to western reptiles and amphibians: Houghton Mifflin Harcourt.

Sterkenburg E, Bahr A, Brandström Durling M, Clemmensen KE, and Lindahl BD. 2015. Changes in fungal communities along a boreal forest soil fertility gradient. New Phytologist 207:1145-1158.

Tererai F, Gaetner M, Jacobs SM, and Richardson DM. 2013. Eucalyptus invasions in riparian forests: effects on native vegetation community diversity, stand structure and composition. Forest Ecology and Management 297:84-93.

van Kleunen M, Dawson W, EssI F, Pergl J, Winter M, Weber E, Kreft H, Weigelt P, Kartesz J, Nishino M, Antonova LA, Barcelona JF, Cabezas FJ, Cardenas D, Cardenas-Toro J, Castano N, Chacon E, Chatelain C, Ebel AL, Figueiredo E, Fuentes N, Groom QJ, Henderson L, Inderjit, Kupriyanov A, Masciadri S, Meerman J, Morozova O, Moser D, Nickrent DL, Patzelt A, Pelser PB, Baptiste MP, Poopath M, Schulze M, Seebens H, Shu WS, Thomas J, Velayos M, Wieringa JJ, and Pysek P. 2015. Global exchange and accumulation of non-native plants. Nature 525:100-+ DOI: 10.1038/nature14910.

Vila M, Espinar JL, Hejda M, Hulme PE, Jarosik V, Maron JL, Pergl J, Schaffner U, Sun Y, and Pysek P. 2011. Ecological impacts of invasive alien plants: a meta-analysis of their effects on species, communities and ecosystems. Ecology Letters 14:702-708 DOI: 10.1111/j.1461-0248.2011.01628.x.

Walke JB, Becker MH, Loftus SC, House LL, Cormier G, Jensen RV, and Belden LK. 2014. Amphibian skin may select for rare environmental microbes. ISME Journal 8:2207-2217 DOI: 10.1038/ismej.2014.77.

Peer) reviewing PDF | (2019:10:42191:1:1:NEW 22 Dec 2019) 
711 Welsh Jr HH, and Droege S. 2001. A case for using plethodontid salamanders for monitoring biodiversity and ecosystem integrity of North American forests. Conservation Biology 15:558-569.

Wolf KM, and DiTomaso JM. 2016. Management of blue gum eucalyptus in California requires region-specific consideration. California Agriculture 70:39-47 DOI: 10.3733/ca.v070n01p39.

Woodhams DC, Brandt H, Baumgartner S, Kielgast J, and Kuepfer E. 2014. Interacting symbionts and immunity in the amphibian skin mucosome predict disease risk and probiotic effectiveness. Plos One 9 DOI: 10.1371/journal.pone.0104590.

Zaneveld JR, McMinds R, and Thurber RV. 2017. Stress and stability: applying the Anna Karenina principle to animal microbiomes. Nature Microbiology 2:17121.

Zhang P, Neher DA, Li B, and Wu JH. 2018. The Impacts of Above- and Belowground Plant Input on Soil Microbiota: Invasive Spartina alterniflora Versus Native Phragmites australis. Ecosystems 21:469-481 DOI: 10.1007/s10021-017-0162-8.

725 
Figure 1

Dominant vegetation map of Wildcat Canyon Regional Park, Tilden Regional Park, and UC Berkeley Campus.

Sampling sites are displayed for Quercus and Eucalyptus dominant habitats from whichBatrachoseps attenuatus skin microbiome swabs and soil were collected. 


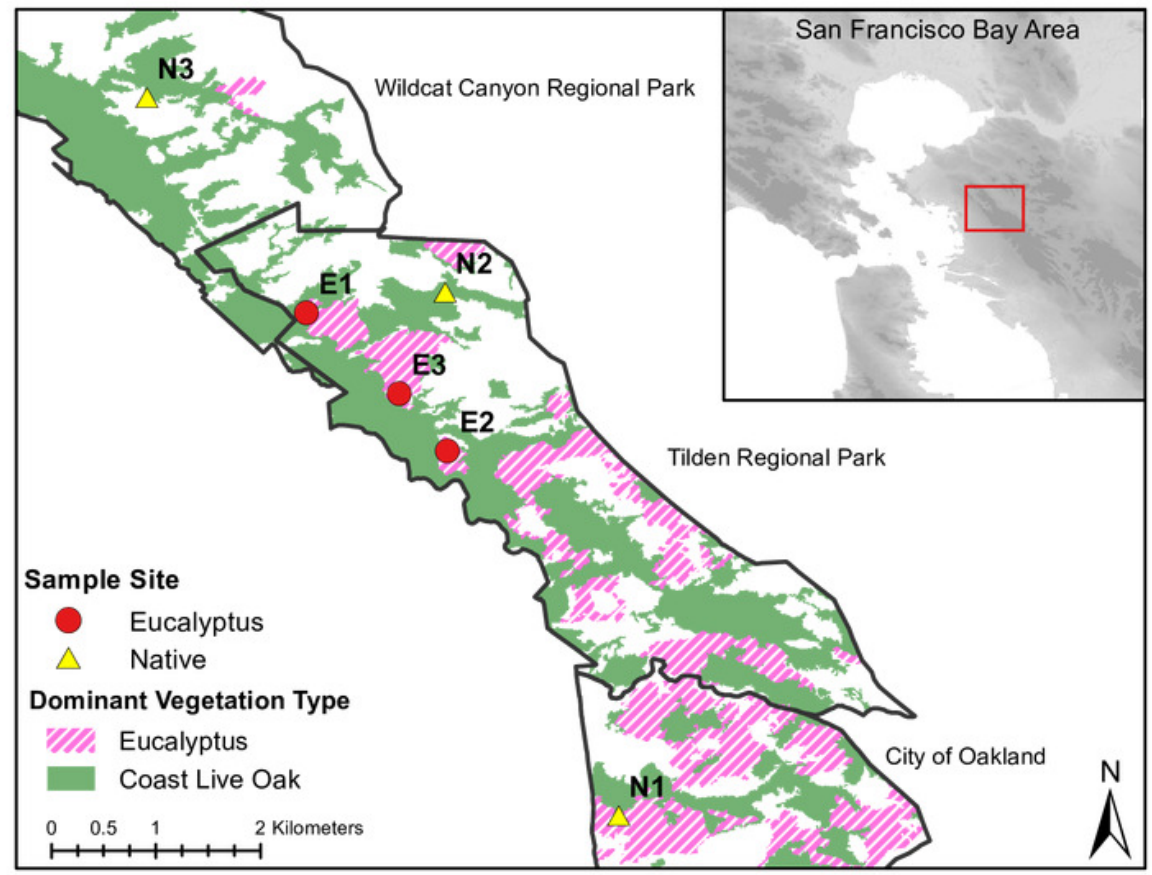


Figure 2

Alpha diversity dot plots with mean (long horizontal line) and standard errors (short horizontal line).

Data is presented for microhabitat soil $(A, B, C)$ and Batrachoseps attenuatus skin $(D, E, F)$ microbiota samples. Alpha diversity measures presented include community richness (A\&D), Shannon Diversity index (B\&E), and Phylogenetic Diversity (C\&F). Each point represents the bacterial skin community of an individual sample; point color indicates dominant vegetation of the habitat (Black - Quercus agrifolia and gray - Eucalyptus globulus) and shape indicates site identity (square - site 1, triangle - site 2, and diamond - site 3 ).
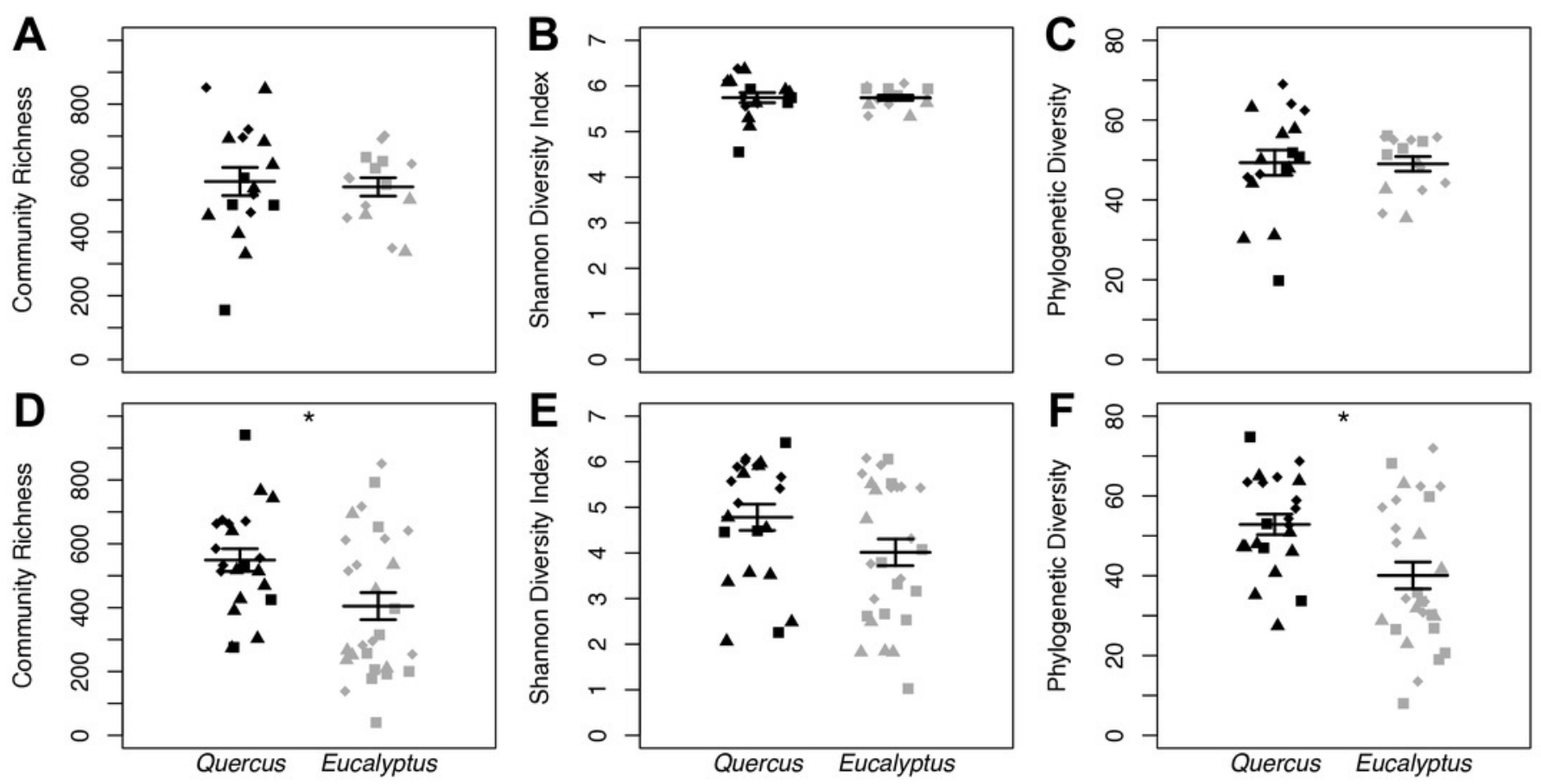
Figure 3

NMDS plots of unweighted UniFrac $(A \& D)$, weighted UniFrac $(B \& E)$, and Bray-Curtis $(C \& F)$ distance matrices from microhabitat soils $(A, B, C)$ and Batrachoseps attenuatus skin microbiota samples (D,E,F).

Each point represents the bacterial skin community of an individual sample; point color indicates dominant vegetation of the habitat (Black - Quercus agrifolia and gray -Eucalyptus globulus) and shape indicates site identity (square - site 1, triangle - site 2, and diamond site 3).
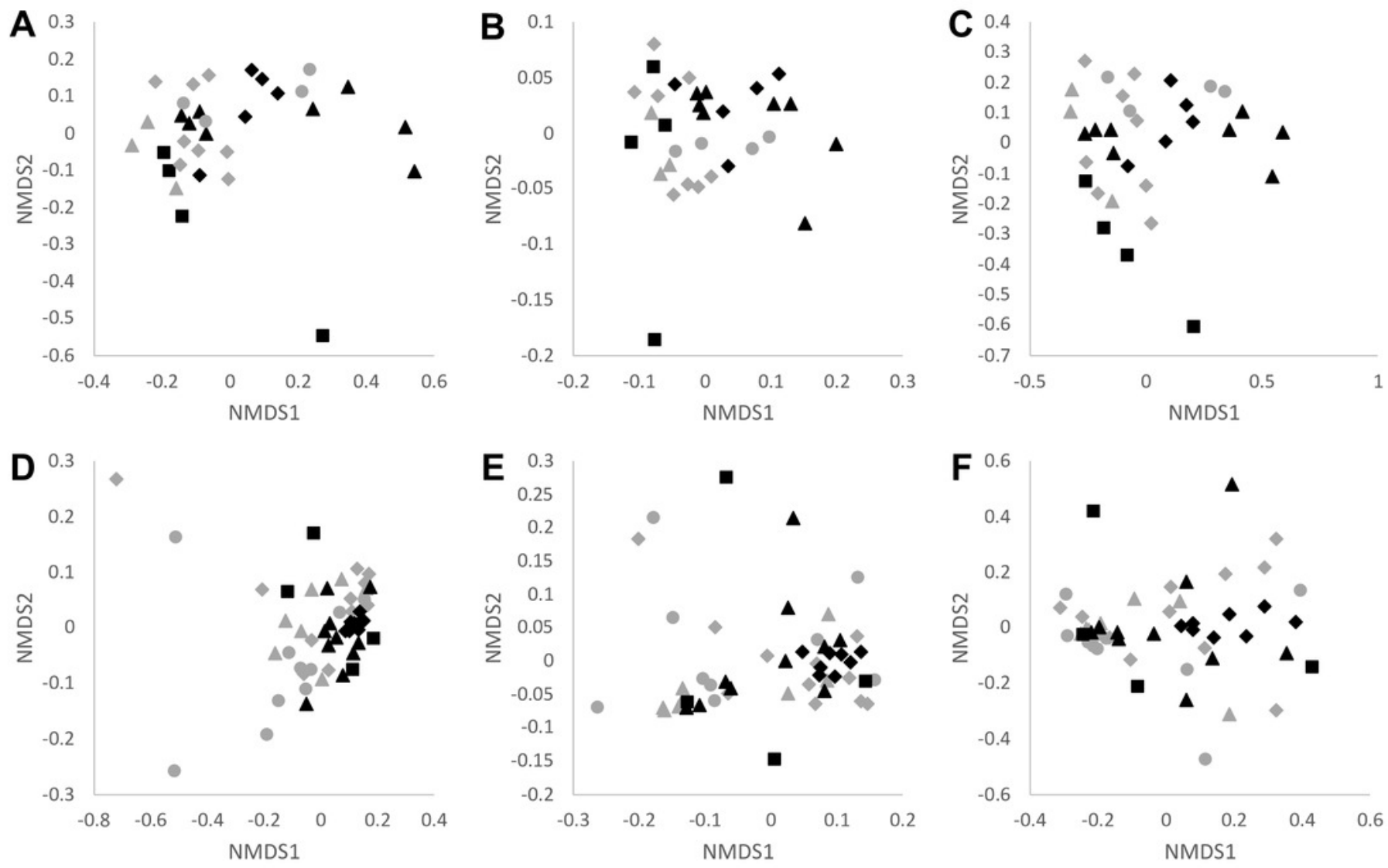
Figure 4

Dot plot of multivariate homogeneity of groups dispersions (betadisper) of Batrachoseps attenuatus skin microbiota samples collected in Quercus agrifolia and Eucalyptus globulus dominant habitats.

Each point represents the bacterial skin community of an individual sample; point color indicates dominant vegetation of the habitat (Black - Quercus agrifolia and gray -Eucalyptus globulus) and shape indicates site identity (square - site 1, triangle - site 2, and diamond site 3).

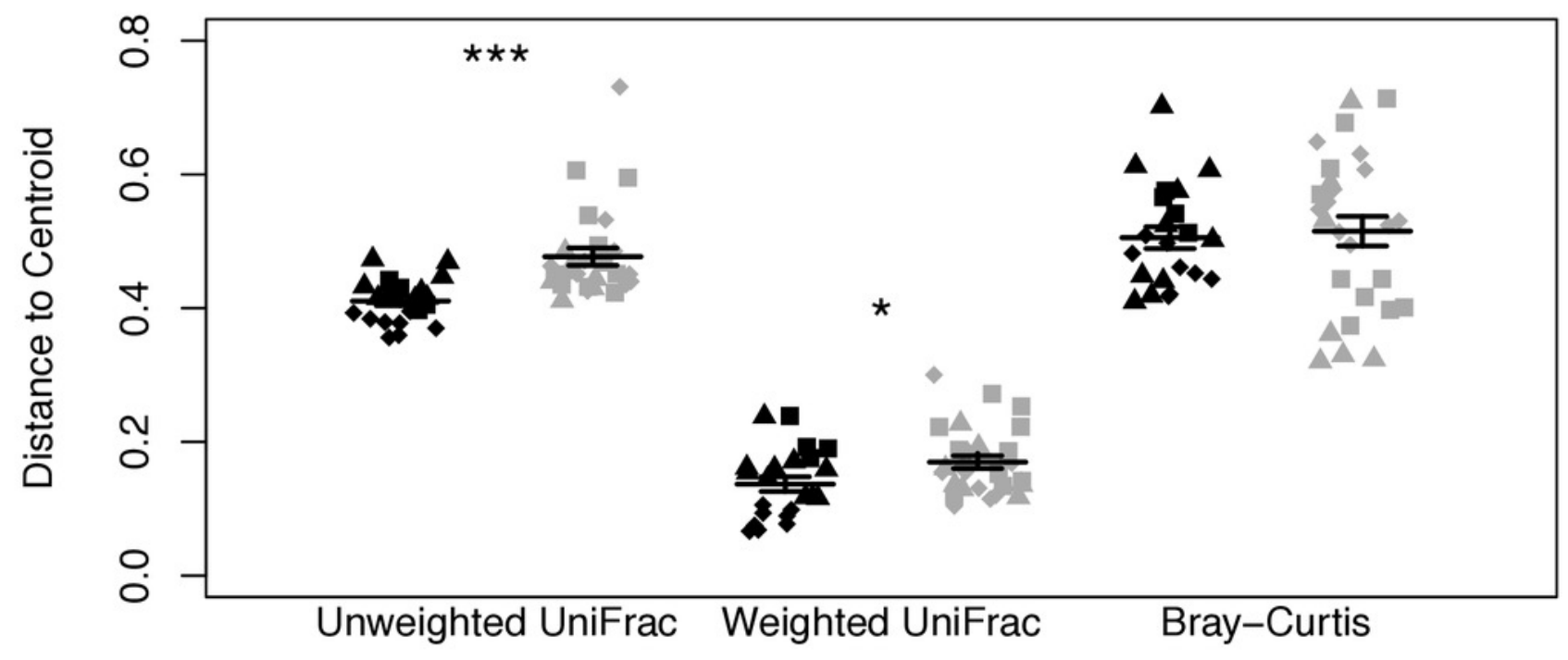


Figure 5

Batrachoseps attenuatus body condition index dot plots with mean (long horizontal line) and standard errors (short horizontal line).

Each point represents the bacterial skin community of an individual sample; point color indicates dominant vegetation of the habitat (Black - Quercus agrifolia and gray -Eucalyptus globulus) and shape indicates site identity (square - site 1, triangle - site 2, and diamond site 3).

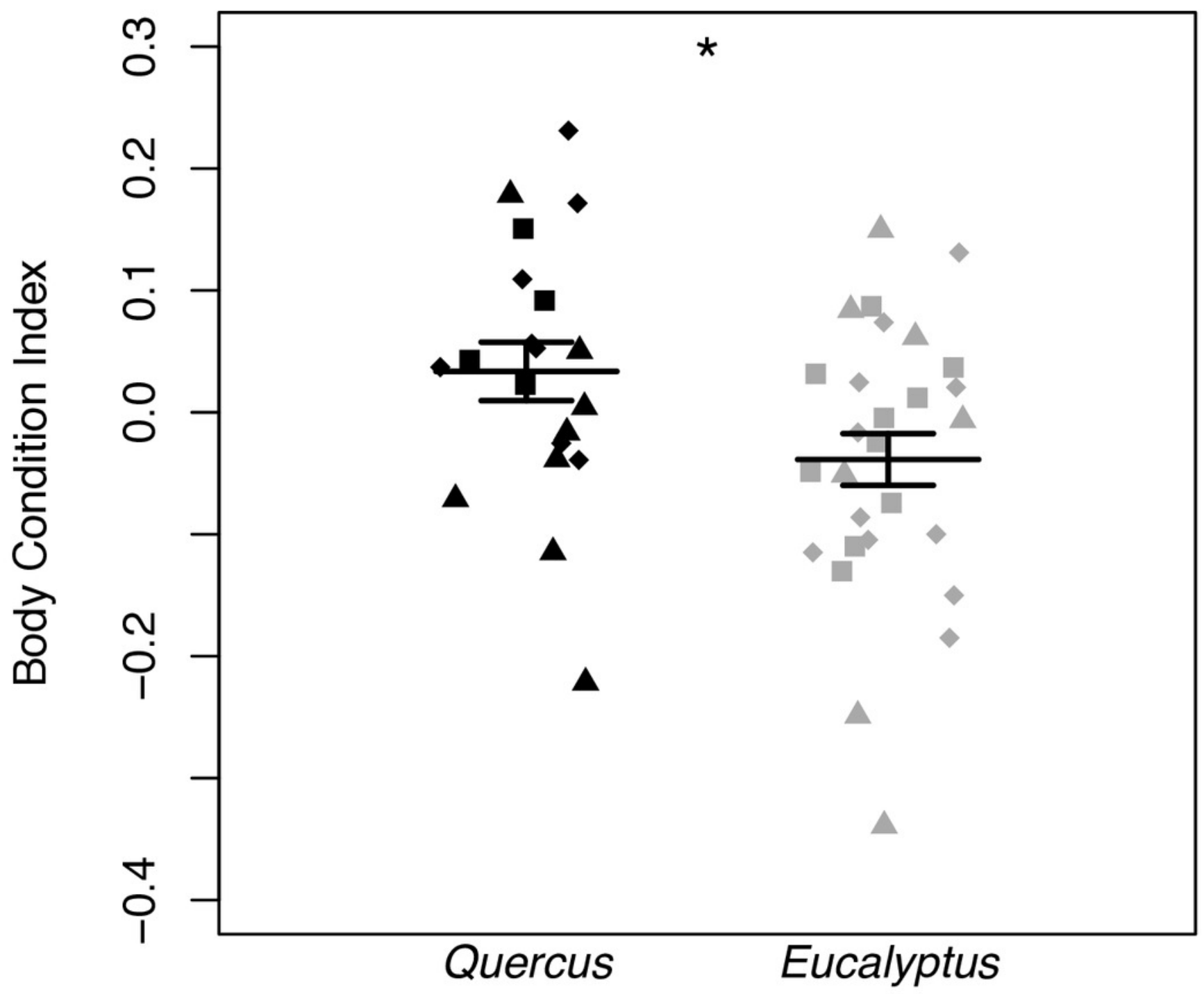

